Article

\title{
Changes in miRNA Expression Profiling during Neuronal Differentiation and Methyl Mercury-Induced Toxicity in Human in Vitro Models
}

\author{
Giorgia Pallocca ${ }^{1,2}$, Marco Fabbri ${ }^{1,3}$, Silvia Nerini-Molteni ${ }^{1}$, Francesca Pistollato ${ }^{1}$, \\ Dimitra Zagoura ${ }^{1}$, Maria Grazia Sacco ${ }^{1}$, Laura Gribaldo ${ }^{1}$, Susanne Bremer-Hoffmann ${ }^{1}$ \\ and Anna Bal-Price ${ }^{1, *}$
}

1 Institute for Health and Consumer Protection, European Commission Joint Research Centre, Ispra (VA) 21027, Italy; E-Mails: giorgia.pallocca@yahoo.it (G.P.); marco.fabbri@gmail.com (M.F.); silvia.nerini-molteni@jrc.ec.europa.eu (S.N.-M.); francesca.pistollato@gmail.com (F.P.); dimitra.zagoura@ec.europa.eu (D.Z.); mariagrazia.sacco@jrc.ec.europa.eu (M.G.S.); laura.gribaldo@ec.europa.eu (L.G.); susanne.bremer-hoffmann@ec.europa.eu (S.B.-H.)

2 Department of Biology, University of Konstanz, Konstanz 78464, Germany

3 Department of Experimental and Clinical Medicine, University of Insubria, Varese 21100, Italy

* Author to whom correspondence should be addressed; E-Mail: anna.price@jrc.ec.europa.eu; Tel.: +39-0332-78-6018; Fax: +39-0332-78-5336.

Received: 4 June 2014; in revised form: 31 July 2014 / Accepted: 5 August 2014 / Published: 29 August 2014

\begin{abstract}
MicroRNAs (miRNAs) are implicated in the epigenetic regulation of several brain developmental processes, such as neurogenesis, neuronal differentiation, neurite outgrowth, and synaptic plasticity. The main aim of this study was to evaluate whether miRNA expression profiling could be a useful approach to detect in vitro developmental neurotoxicity. For this purpose, we assessed the changes in miRNA expression caused by methyl mercury chloride $(\mathrm{MeHgCl})$, a well-known developmental neurotoxicant, comparing carcinoma pluripotent stem cells (NT-2) with human embryonic stem cells (H9), both analyzed during the early stage of neural progenitor commitment into neuronal lineage. The data indicate the activation of two distinct miRNA signatures, one activated upon neuronal differentiation and another upon $\mathrm{MeHgCl}$-induced toxicity. Particularly, exposure to $\mathrm{MeHgCl}$ elicited, in both neural models, the down-regulation of the same six out of the ten most up-regulated neuronal pathways, as shown by the up-regulation of the corresponding miRNAs and further assessment of gene ontology (GO)
\end{abstract}


term and pathway enrichment analysis. Importantly, some of these common miRNA-targeted pathways defined in both cell lines are known to play a role in critical developmental processes, specific for neuronal differentiation, such as axon guidance and neurotrophin-regulated signaling. The obtained results indicate that miRNAs expression profiling could be a promising tool to assess developmental neurotoxicity pathway perturbation, contributing towards improved predictive human toxicity testing.

Keywords: miRNAs expression profiling; NT-2 and H9 cell lines; neuronal cultures; pathways of toxicity

\section{Introduction}

Neuronal gene expression is tightly regulated during development of central nervous system (CNS). Indeed, the process of neurogenesis requires the coordinated up regulation of neuronal genes and down-regulation of non-neuronal genes to ensure the proper timing of differentiation and correct number of neurons. Recently, a great deal of evidence showed that gene expression regulation includes, not only transcription factors, but also small regulatory RNAs, such as microRNAs (miRNAs), which play an important role in the establishment and maintenance of cell identity, neuronal and glial differentiation, axonal path-finding, neuronal patterning, and plasticity [1-3]. miRNAs introduce an additional layer of post-transcriptional regulatory control of gene expression since each miRNA can target up to a few hundred target genes, resulting in the regulation of as much as one third of the protein expression in animals [4].

The role of miRNAs in brain development has been studied by analyzing the effects of the genomic Dicer ablation, which results in the absence of all mature miRNAs. In mice, Dicer ablation causes neurodegeneration of dopaminergic neurons in the midbrain [5] and Dicer silencing in neocortical progenitors impairs neuronal differentiation [6] and results in abnormal terminal differentiation of developing olfactory progenitors. Severe brain morphogenesis deficits were also observed in zebrafish Dicer knockout mutants [7].

Several miRNAs have been found to play important roles in the nervous system. The brain specific miRNA-9 targets the stathmin mRNA and has been implicated in early neurogenesis, promoting cell proliferation while decreasing neural progenitors migration $[8,9]$. Another brain specific miRNA is miRNA-124, known to be involved in neuronal differentiation [10].

Numerous miRNAs, including miRNA-134, have been reported to play an important role in modulation of spines and dendrites development [11] and others, such as miRNA-132 [12] and miRNA-388, affect neurite outgrowth, branching synaptic plasticity, and memory function [1]. There are a few identified miRNAs (miRNAs-284, miRNA-138, miRNA-1) that have been implicated in synaptic formation and plasticity [1], as well as in the establishment of neuronal connectivity (such as miRNA-206, let-7 or miRNA-125a), which plays an important role in learning and memory processes $[13,14]$. The above-mentioned studies suggest that miRNAs are heavily involved in the regulation of neuronal development at different stages of cell differentiation and maturation. Moreover, it is well known that abnormal miRNA expression resulting in aberrant expression of target 
mRNAs can take place during neurodegenerative disorders or as a result of chemically induced neurotoxicity [15]. For instance, miRNA-19, -101, and -130 have been shown to regulate the expression of genes involved in spino-cerebellar ataxia [16] and miRNA-107 is involved in the pathophysiology of Alzheimer's disease [17]. The main aim of this study was to evaluate whether the analysis of the dysregulation of miRNA expression profiles induced by the exposure to an environmental chemical, could be a useful tool for developmental neurotoxicity testing (DNT) using an in vitro approach. Therefore, to identify the possible DNT biomarkers among deregulated miRNAs, we have firstly studied the changes in miRNA expression during the process of neuronal differentiation in the neuronal cultures derived from H9 or NT-2 cell lines and then compared the miRNA expression profiles in the same cell lines observed after exposure to a non-cytotoxic concentration of methyl mercury chloride $(\mathrm{MeHgCl}) . \mathrm{MeHgCl}$ is a well-known DNT chemical that disturbs the process of neuronal differentiation, synapse formation and causes damage to the process of learning and memory [18-20]. The exposure to $\mathrm{MeHgCl}$ was performed during the early stages of the neuronal differentiation process (i.e., 1-36 days in vitro (DIV) for NT-2 cells and 1-10 DIV for H9 cells) since previous in vitro studies from our laboratory [19,20] and epidemiological evidence [21-24] indicate that, compared to the adult CNS, the early stages of brain development are more susceptible to toxicants. Moreover, the early exposure of neural progenitor cells to chemicals seems to be a more vulnerable time period than the later stages of neuronal differentiation [25,26]. Indeed, human embryonic stem cells (hESCs), at the stage of the neural precursor formation, have been shown to be affected by non-cytotoxic concentrations of $\mathrm{MeHgCl}$ [26,27].

In these studies we have compared the miRNA expression profile of the control neuronal cultures derived from two different sources of human stem cells, pluripotent embryonic carcinoma (NT2 cell line) with embryonic stem cells (H9 cell line) both assessed against their respective undifferentiated and differentiated counterparts. At the next stage, the changes in the expression of miRNA expression were evaluated after exposure to $\mathrm{MetHgCl}$, possibly contributing to the identification of novel biomarkers for neurodevelopmental toxicity, implementing the knowledge of the molecular mechanisms implicated in human toxicology.

Currently, different types of human stem cell models are widely used for basic research, regenerative medicine and toxicological studies; however, works addressing comparative analyses of the proposed models, especially for toxicological applications, are mostly missing. Recently, human pluripotent stem cells (hPSCs) and their derivatives have been considered as valuable cellular tools to implement in current toxicity testing [28-30]. Particularly, these cells can be efficiently differentiated towards several cell types, including neuronal and glial-like cells, representing valid tools to study both developmental and adult neurotoxicity [29,31].

As characterized in our previous studies, the human pluripotent carcinoma stem cell line NT2 cell line, in the presence of retinoic acid (RA), can be differentiated into post-mitotic neurons expressing MAP2, NF200, neuronal class Tub-ßIII, MAPT-Tau, synaptophysin, and other proteins [32]. Furthermore, under specific culture conditions, the NT2-derived neurons can form functional synapses [33] and express a variety of synapse formation markers and neurotransmitters phenotypes [34]. Similarly, the H9 cell line can be committed toward neural progenitors [26] and further differentiated towards neuronal and glial phenotype $[29,35]$. 
In this study, we have analyzed the mRNA and miRNA expression profiles comparing NT-2 and H9 cells undergoing early neural commitment, followed by the evaluation of the effects elicited by a repeated administration of $\mathrm{MeHgCl}$. Data indicate the downregulation of common neuronally related signaling pathways in both cellular models, as highlighted by miRNAs/mRNA gene ontology analyses. This knowledge might contribute to the development of a new class of toxicological in vitro tests, leading to the design of a novel mechanistic toxicology, based on human in vitro models. In this context, miRNA profiling could provide functional information on cellular physiological pathways that when significantly perturbed by the exposure to a chemical, might be acknowledged as toxicity pathways involved in developmental neurotoxicity.

\section{Materials and Methods}

\subsection{H9 and NT-2 Cell Maintenance and Differentiation towards Neuronal Phenotype}

Both types of pluripotent stem cells were triggered to differentiate into neuronal-like cells following well-established protocols. Undifferentiated human embryonic stem cells (H9, hESCs) (WiCell MOU 02-W177 SLA 10-W0409) were cultured and committed toward neural precursor cells for 10 DIV, followed up to full post-mitotic neuronal differentiation (28 DIV) as previously described [26]. Briefly, NIH-registered $\mathrm{H} 9$ cells were cultured at $37{ }^{\circ} \mathrm{C}$ and $5 \% \mathrm{CO}_{2}$ in maintenance medium (DMEM/F12 supplemented with $20 \%$ knock out serum replacement, $1 \%$ non-essential amino acids, $50 \mathrm{U} / \mathrm{mL}$ penicillin and $50 \mu \mathrm{g} / \mathrm{mL}$ streptomycin, $2 \mathrm{mM}$ glutamine, $0.1 \mathrm{mM} \beta$-mercaptoethanol (all from Invitrogen, Carlsbad, CA, USA) and $8 \mathrm{ng} / \mathrm{mL}$ of human basic fibroblast growth factor (bFGF, Immunological Sciences, Rome, Italy). H9 cells were sub-cultured weekly by microdissection and propagated on mitomycin-inactivated human feeder fibroblasts (hDFn, Gibco, Cascade Biologics, Carlsbad, CA, USA), which have been plated on 0.1\% gelatin (Sigma Aldrich, St. Louis, MO, USA), diluted in $1 \times$ PBS (Gibco, Life Technologies, Carlsbad, CA, USA) at a plating density of about 15,000 cells $/ \mathrm{cm}^{2}$.

Further differentiation of neural precursors was performed as follows: intact $\mathrm{H} 9$ colonies were detached by $1 \mathrm{mg} / \mathrm{mL}$ collagenase (Invitrogen, Carlsbad, CA, USA) and cultured in suspension in maintenance medium without bFGF to allow embryoid body (EB) formation. Each EB was transferred onto a single well in $10 \mu \mathrm{g} / \mathrm{mL}$ laminin-coated black, clear-bottom 96-well plates (Perkin Elmer ViewPlate-96F Cat No. 6005225, Waltham, MA, USA), containing neural induction (NI) medium (DMEM/F12 supplemented with 1\% nonessential amino acids, 1\% N2 supplement (Invitrogen, Carlsbad, CA, USA), and $2 \mu \mathrm{g} / \mathrm{mL}$ heparin (Sigma, St. Louis, MO, USA) and $20 \mathrm{ng} / \mathrm{mL} \mathrm{bFGF.}$ Cultures were observed daily and medium was changed every third day for 10 days. During this time, neuroepithelial aggregates, called rosettes, became visible. To promote the full maturation of the neuroepithelial cells into mature neurons, rosettes were cut into fragments using a $30 \mathrm{G}$ needle and p100 $\mu \mathrm{L}$ tip, using a phase contrast microscope under sterile conditions. Fragments were replated on laminin-coated dishes in presence of neuronal differentiation medium (ND, Neural progenitor cell basal medium, supplemented with NPDM Bulletkit, $1 \mathrm{ng} / \mathrm{mL}$ GDNF and $2.5 \mathrm{ng} / \mathrm{mL}$ BDNF) and cultured for at least 3 additional weeks. 
NTERA-2/cl.D1 cells were differentiated into neurons as previously described in [32]. Briefly, undifferentiated NT-2 cells (ATCC) were cultured in standard tissue culture flasks (Nunc, Rochester, NY, USA) and maintained in Opti-MEM I (Gibco, Carlsbad, CA, USA) supplemented with 5\% fetal bovine serum (FBS) (HyClone, Waltham, MA, USA), $100 \mathrm{U} / \mathrm{mL}$ penicillin (P), and $100 \mu \mathrm{g} / \mathrm{mL}$ streptomycin (S) (Gibco, Carlsbad, CA, USA). The differentiation process was performed by culturing the cells in Dulbecco's modified Eagle's Medium-High Glucose (DMEM-HG) (Gibco, Carlsbad, CA, USA) supplemented with $10 \% \mathrm{FBS}, 1 \% \mathrm{P} / \mathrm{S}$, and $10 \mu \mathrm{M}$ retinoic acid (RA) (Sigma, St. Louis, MO, USA) up to $36 \mathrm{DIV}$ at $37{ }^{\circ} \mathrm{C}$ in a humidified atmosphere of $5 \%$ of $\mathrm{CO}_{2}$. To obtain fully differentiated neurons, the cells were trypsinized at $36 \mathrm{DIV}$, and split in standard medium (DMEM-HG with $5 \%$ FBS, P/S) supplemented with a mixture of mitosis inhibitors composed of $1 \mu \mathrm{M}$ cytosine arabinoside (Sigma, St. Louis, MO, USA), $10 \mu \mathrm{M}$ fluorodeoxyuridine (Sigma, St. Louis, MO, USA), and $10 \mu \mathrm{M}$ uridine (Sigma, St. Louis, MO, USA). Cells were then seeded in plates coated with $10 \mu \mathrm{g} / \mathrm{mL}$ poly-D-lysine (Sigma, St. Louis, MO, USA) and $0.26 \mathrm{mg} / \mathrm{mL}$ Matrigel and cultured for the next 8 weeks (up to $96 \mathrm{DIV}$ ) at $37{ }^{\circ} \mathrm{C}$ in a humidified atmosphere of $5 \%$ of $\mathrm{CO}_{2}$. The medium was changed twice per week.

\subsection{Immunocytochemistry}

Fully differentiated H9 cells (28 DIV) and fully differentiated NT-2 cells (96 DIV) were fixed with $4 \%$ formaldehyde (Sigma, St. Louis, MO, USA) for $20 \mathrm{~min}$ at room temperature. After three washes with PBS buffer (Invitrogen, Carlsbad, CA, USA), the cells were permeabilized for 15 min with 0.4\% Triton X-100 (Sigma, St. Louis, MO, USA). Blocking was done using 3\% of BSA (Sigma, St. Louis, MO, USA) in PBS for 30 minutes at room temperature. The blocking buffer was removed by aspiration and replaced by primary antibodies against the neural stem cell marker nestin (Sigma, St. Louis, MO, USA, rabbit, 1:200) or neurofilament 200 (NF-200, Sigma, St. Louis, MO, USA, rabbit, 1:1000), diluted in 3\% BSA-blocking buffer. Plates were incubated for $1 \mathrm{~h}$ at $37{ }^{\circ} \mathrm{C}$ or overnight at $4{ }^{\circ} \mathrm{C}$. Stained cells were then rinsed with $1 \times$ PBS buffer and further incubated for $30 \mathrm{~min}$ at room temperature with the appropriate fluorescent-conjugated secondary antibodies (Alexa Fluor-488 goat anti-rabbit IgG $(\mathrm{H}+\mathrm{L}), 1: 1000$, and Alexa Fluor-546 goat anti-mouse $\operatorname{IgG}(\mathrm{H}+\mathrm{L})$, 1:1000) and nuclei were counterstained by using DAPI dye $(0.3 \mu \mathrm{M}$, Sigma, St. Louis, MO, USA). Controls for specificity of immunostaining were performed by omitting the primary antibody from the procedure. All stained cultures were examined by fluorescent microscope (Olympus $1 \times 70$, Tokyo, Japan).

\subsection{Cell Viability Assay Using Alamar Blue (AB)}

Cell viability was determined once a week during the five-week-long treatment of NT-2 cells with 100, 200, 400 and $500 \mathrm{nM} \mathrm{MeHgCl}$, using the $\mathrm{AB}$ (resazurine) assay [36]. The blue colored indicator dye resazurin is reduced into fluorescent resorufin by red-ox reactions in viable cells. Resazurin $(10 \mu \mathrm{L}$ of $100 \mu \mathrm{M}$ stock) in Hank's Buffered Salt Solution was added directly to the 96-well plates, without removing the medium $(100 \mu \mathrm{L})$. The plate was incubated for $6 \mathrm{~h}$ at $37{ }^{\circ} \mathrm{C}, 5 \% \mathrm{CO}_{2}$. After the incubation period, the fluorescence of the resazurin metabolite (resorufin) was measured at 
$530 \mathrm{~nm} / 590 \mathrm{~nm}$ (excitation/emission) in a multi-well fluorometric reader (Tecan i-control, Shanghai, China). The results were expressed as a percentage of the mean value for the untreated cultures.

The same $\mathrm{AB}$ assay was performed on differentiated H9 cells only once after the 10 days of exposure to $25 \mathrm{nM} \mathrm{MeHgCl}$, in order to ensure that it was not a cytotoxic concentration.

\subsection{Exposure to Methyl Mercury Chloride}

Neural differentiating hESCs (H9 cell line) were exposed to a non-cytotoxic concentration (based on Alamar Blue assay, data not shown) of $\mathrm{MeHgCl}$ (25 nM, Sigma, St. Louis, MO, USA). Chemical exposure began $24 \mathrm{~h}$ after EB plating (day 1 of differentiation). Both chemical and medium were refreshed every third day along the neural commitment process, allowing a repeated exposure to the chemical. Exposure lasted 10 days; on day 10, samples were harvested for mRNAs and miRNAs analyses.

Analogously, NT-2 cells were exposed to a non-cytotoxic concentration (based on Alamar Blue assay, [36]) of $\mathrm{MeHgCl}$ (400 nM, Sigma, St. Louis, MO, USA) from 2-36 DIV during the cell differentiation process and both medium and chemical treatment were refreshed twice a week. Three biological replicates were performed per each experimental condition (control and treated cells).

\subsection{RNA Extraction and miRNAs Expression Analysis}

RNA was extracted from untreated and $\mathrm{MeHgCl}$ treated cell samples using the MIRVANA kit (AMBION, Foster City, CA, USA) according to manufacturer's instructions. This kit allows the isolation of total RNA enriched with miRNAs. RNA concentration and quality were determined by Nanodrop (Thermo Fisher Scientific, Boston, MA, USA) and 2100 Agilent Bioanalizer (Agilent Genomics, Santa Clara, CA, USA). The RIN was always above 8.5.

Total RNA was reverse transcribed with Taqman MicroRNA Reverse Transcription Kit using Megaplex $^{\mathrm{TM}}$ RT Primers (Applied Biosystems, Carlsbad, CA, USA). Real Time PCR reactions were carried out on pre-configured microfluidic cards (Taqman Array MicroRNA Cards, set A,V2.2, Applied Biosystems, Carlsbad, CA, USA) allowing the detection of about specific 380 unique assays and four candidate endogenous control assays. The microfluidic cards were evaluated with Applied Biosystems 7900HT Sequence Detection system (Applied Biosystems, Carlsbad, CA, USA). For H9 cell line, two independent RNA samples per group were obtained by pooling 16 biological replicates (i.e., individual EBs pooled together); for NT-2 cell line, three biological replicates were performed for every experimental condition.

\subsection{Analysis of Stemness Marker Expression}

The expression of a set of genes known as stemness markers specific for hESCs was evaluated using TaqMan ${ }^{\circledR}$ Human Stem Cell Pluripotency microfluidic cards (Applied Biosystems, Carlsbad, CA, USA). The expression of 90 well-defined genes validated as markers for pluripotency and differentiation, together with 6 endogenous controls, was evaluated after retro-transcription of total RNA in cDNA by using the High Capacity cDNA reverse Transcription Kit (Applied Biosystems, Carlsbad, CA, USA). The microfluidic cards were analysed with Applied Biosystems 7900HT 
Sequence Detection system (Applied Biosystems, Carlsbad, CA, USA). Each measurement was performed in two independent experiments for NT-2 and H9 cell lines, two independent RNA samples per group were obtained by pooling 16 biological replicates.

\subsection{Statistical Analysis of miRNA Expression}

Experimental data were then analyzed by SDS 2.3 software (Applied Biosystems, Carlsbad, CA, USA). The Ct values were exported from SDS 2.3 software (Applied Biosystems, Carlsbad, CA, USA) and used as raw data for analysis of qRTPCR data. The R software (version 3.0.2, Vienna, Austria, 2013) [37] and the packages HTqPCR [38] and Limma [39] were used for the manipulation and analysis of the $C t$ values.

Statistical significance was assessed using Limma (linear models in micro-array analysis) in HTqPCR. Statistical comparisons were generated for differentiated neuronal cultures samples between the treated and control samples. Detectors that showed a fold change greater than 2 or less than 0.5 , with a $p$-value smaller than 0.05 , were considered as differentially expressed.

\subsection{Gene Ontology (GO) Term Enrichment Analysis}

The miRecords database [40] was used to identify the predicted targets for regulated microRNA that were further analyzed for identification of pathways enrichment.

MiRecords stores predicted miRNA targets produced by 11 established miRNA target prediction programs (DIANA-microT, MicroInspector, miRanda, MirTarget2, miTarget, NBmiRTar, PicTar, PITA, RNA22, RNAhybrid, and TargetScan.). In the miRecords database, we considered as predicted when they were identified as targets by at least five different algorithms.

The targets were organized in three lists: targets of up-regulated miRNA in $\mathrm{H} 9$ cells by $\mathrm{MeHgCl}$ treatments, targets of up-regulated miRNA in NT-2 cells after $\mathrm{MeHgCl}$ treatments and targets of down-regulated miRNA in NT-2 cells after exposure to $\mathrm{MeHgCl}$.

The further analysis of the three gene lists was conducted online using WEB-based GEne SeT AnaLysis Toolkit (WebGestalt) [14]. For term enrichment analysis defaults settings were applied.

\section{Results}

\subsection{Characterization of H9 and NT2 Cells Differentiation towards Neuronal Phenotype}

The H9 human pluripotent embryonic stem cell line and the NT-2 pluripotent human testicular embryonic carcinoma cell line were differentiated towards the neuronal lineage using two different protocols (see Materials and Methods, section 2.1). Rosette formation in H9 cell culture was observed by phase contrast microscope between 8-10 DIV (Figure 1A). After 28 DIV, the presence of differentiated neuronal-like cells, creating a dense network of neurites, was confirmed by staining with the neuronal differentiation marker NF-200 (Figure 1B). The detailed characterization of neuronal differentiation kinetics of $\mathrm{H} 9$ cell line was previously described [26]. 
Figure 1. Neural differentiation verified by phase-contrast microscope and immuno-cytochemistry staining. (A) Differentiation process triggered the formation of neural rosettes in $\mathrm{H} 9$ cell culture (at passage 35), already detectable on 10 DIV (phase contrast, magnification 10×); (B) At 28 DIV a dense network of neurites was visualized in H9 cell line by staining against NF-200 (green) and DAPI (blue) (fluorescent microscopy) (at passage 35, magnification $10 \times$ ). The table presents the process of neuronal differentiation (the applied media, differentiation factors and type of coating; NI-Neural Induction medium, ND-Neuronal Differentiation medium); (C) The differentiation towards neuronal phenotype of NT-2 cell line was triggered by the exposure to $10 \mu \mathrm{M}$ retinoic acid up to $36 \mathrm{DIV}$ (phase contrast, magnification 20×); (D) The process of neuronal differentiation of NT2 cells was followed up to 96 DIV. At this time point, groups of neurons (nuclei stained by DAPI, blue) were linked by a dense network of neurites, positively stained against NF-200 (green, fluorescent microscopy, magnification $40 \times$ ). The table presents the process of neuronal differentiation (the applied media, differentiation factors, and type of coating; NI-Neural Induction medium, ND-Neuronal Differentiation medium).

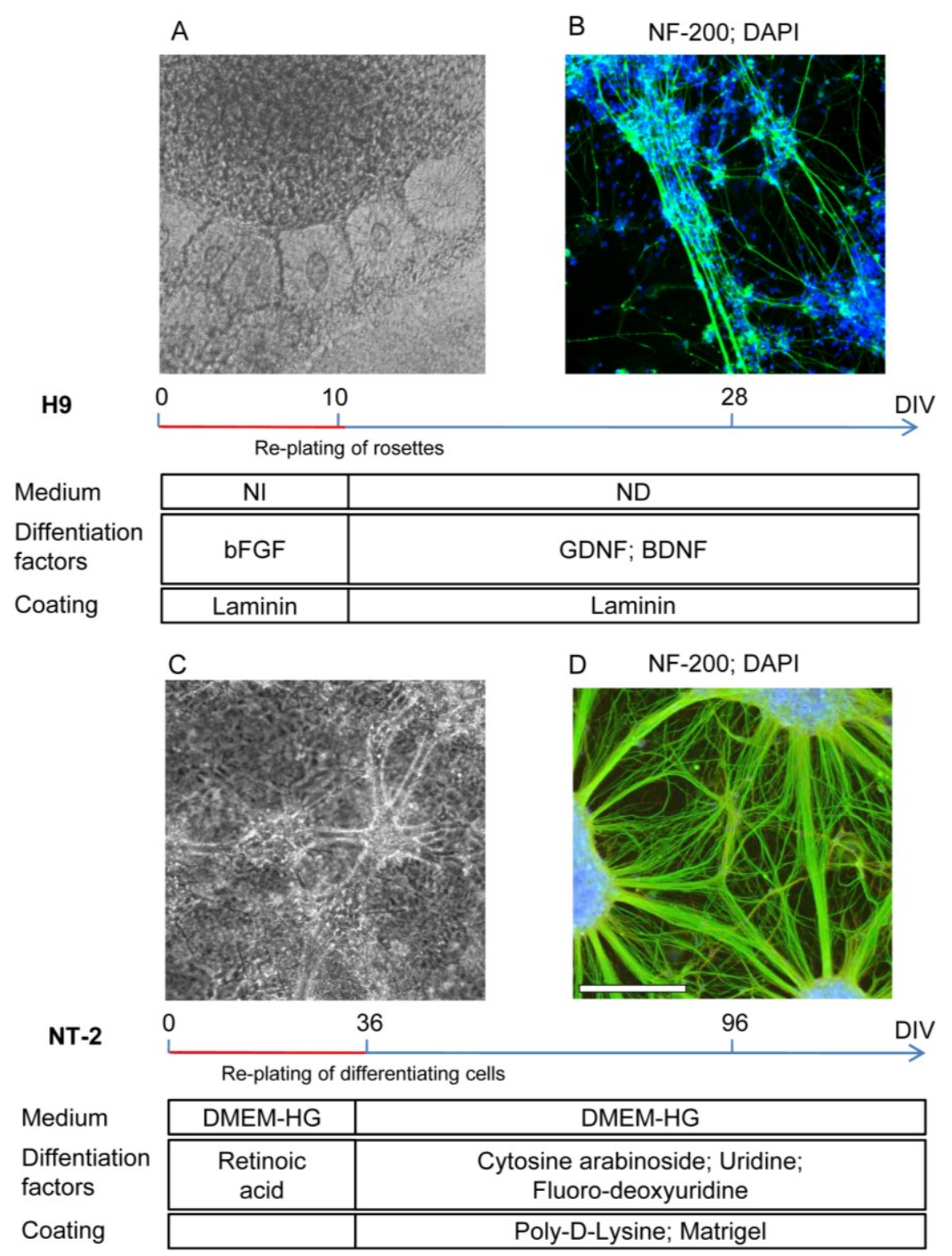


The NT-2 pluripotent human testicular embryonic carcinoma cell line was differentiated toward neuronal phenotype following the procedure described in our previous studies [32]. For the purpose of this work we tried to match the first stage of cell differentiation between these two models and focused only on the initial process of neural precursors differentiation. According to our data sets, the first 10 days of differentiation of $\mathrm{H} 9$ cells in the presence of NI medium corresponded approximately to 36 days of differentiation of NT-2 cells in the presence of RA. Indeed, rosette-like structures of H9 cell line at 10 DIV possessed cells predominantly nestin positive indicating the presence of neural progenitors (data not shown). Similarly, NT-2 cells at 36 DIV (in the presence of RA) were also mainly positive for nestin (data not shown) confirming the presence of neural precursor cells committed to neuronal lineage. Based on these results, we considered NT-2 cells at 36 DIV and H9 cell line at 10 DIV being at similar stage of differentiation, consisting mainly of neural progenitor cells (nestin-positive cells). After 36 DIV in the presence of RA, NT-2 cells started to form a very dense multilayered neuronal culture (Figure 1C). After this stage, the exposure to mitosis inhibitors (until 96 DIV) allowed to obtain a fully matured, mixed neuronal/glial population of cells characterized in our previous studies [32]. At 96 DIV the presence of large cell aggregates was observed, which were linked to each other by neurite bundles, forming a dense neuronal network, positively stained for NF-200 (Figure 1D). The process of neuronal differentiation was followed up to 96 DIV and up to 28 DIV in the case of NT-2 and H9 cell lines, respectively. The final stage of post-mitotic neuronal differentiation was found to be comparable between NT-2 derived neuronal-like cells and H9-derived neuronal culture, as shown by cell morphology based on staining against NF-200 for both cell lines (Figure 1B,D) and expression of specific neuronal markers at mRNA and protein levels [32,35].

\subsection{Gene Expression Analysis Comparing Neuronal Committed H9 and Neuronal Committed NT-2 Cells}

In order to compare the early stage of the differentiation process, the expression levels of 90 different genes were analyzed using the "TaqMan Human Stem Cell pluripotency microfluidic card" in the two cell lines. This card allows the simultaneous analysis of genes validated as characteristic markers of human stem cells or of the three embryonic germ layers. Based on the performed analysis, the overexpression of 27 and 28 genes was identified, respectively in the differentiated H9 (DIV 10) and NT-2 cells (DIV 36). Among these genes, five were significantly up-regulated in both cell lines (COL2A1, FN1, GATA6, IAPP and SYP) compared to their respective undifferentiated counterparts (Figure 2A,B).

The zinc finger transcription factor GATA6 plays a crucial role during early embryogenesis of vertebrate development; the alpha-1 chain of type II collagen (COL2A1) is defined as a mesodermal marker; fibronectin $(F N 1)$ is involved in cell adhesion and migration processes, including embryogenesis; the islet amyloid polypeptide $(I A P P)$ is expressed during the embryonic development of the pancreas; synaptophysin $(S Y P)$ is an integral membrane protein of small synaptic vesicles found in brain and endocrine cells.

In contrast, 29 and 28 genes were down-regulated upon neuronal commitment respectively in $\mathrm{H} 9$ and NT-2 cells. Amongst these, six genes (i.e., DNMT3B, EOMES, FGF4, LIN28, SFRP2 and SOX2) were down-regulated in both cell lines (Figure 3A,B). 
Figure 2. Comparison of mRNA expression of up-regulated stemness phenotype markers in H9 and NT-2 cells upon neural differentiation. (A) The comparison between the number of up-regulated genes in H9 at 10 DIV (blue) and in NT-2 at 36 DIV (red) cell line (compared to undifferentiated cells) illustrated by the use of Venn diagrams. Among the upregulated genes five of them (COL2A1, FN1, GATA6, IAPP, and SYP) were overlapping being over-expressed in both cell systems; (B) The fold change of the gene expression of the five up-regulated genes overlapping between the differentiated H9 and NT2 cell cultures has been quantified by using $\operatorname{TaqMan}^{\circledR}$ Human Stem Cell Pluripotency microfluidic cards.

(A)
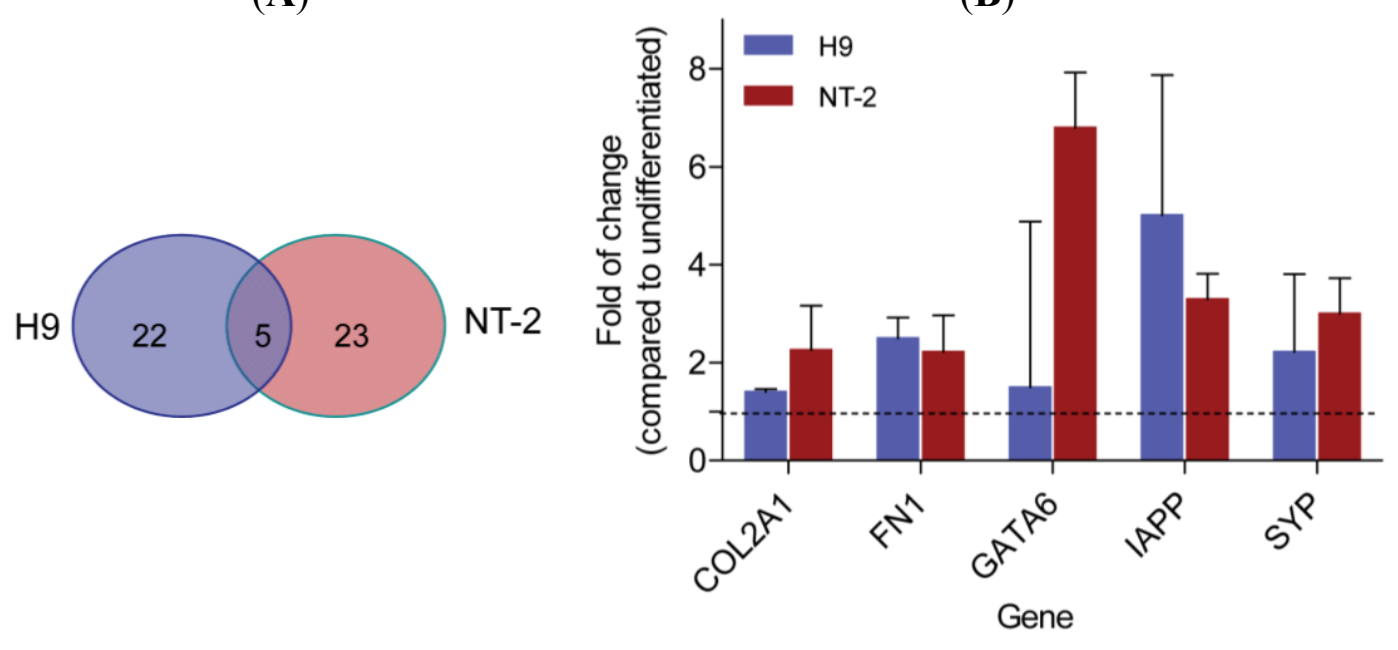

(B)

Figure 3. Comparison of mRNA expression of down-regulated stemness phenotype markers in H9 and NT-2 cells upon neural differentiation. (A) The genes expression overlapping between H9 and NT-2 cell lines represented by the use of Venn diagrams, showing the number of down-regulated genes in H9 (green) and in NT-2 (yellow); (B) The expression of the same six genes was down-regulated in both cell systems (H9 and NT-2) when compared with their fold change to the expression level in undifferentiated cell cultures by using TaqMan ${ }^{\circledR}$ Human Stem Cell Pluripotency microfluidic cards.

(A)

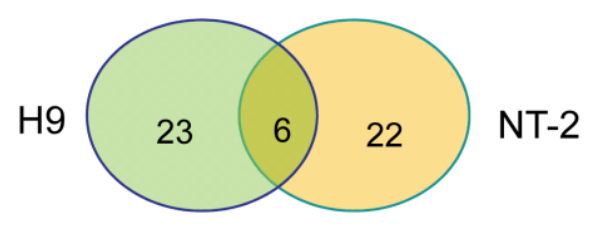

(B)

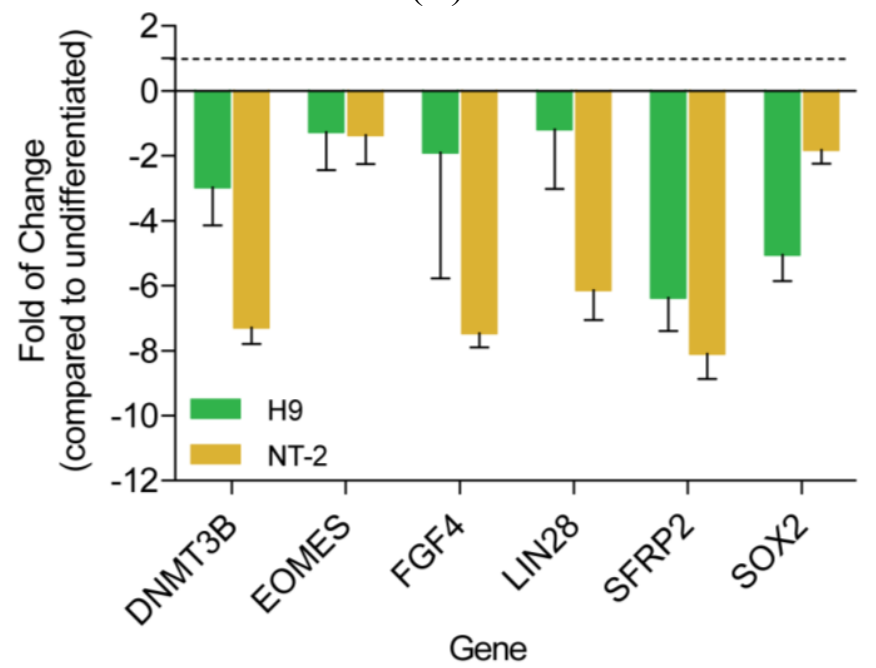


With the exception of EOMES, a trophoblast marker, other genes were correlated to stemness properties and phenotype. DNMT3B, a DNA methyltransferase, is known to play an important role in embryonic development of undifferentiated hESCs; the transcription factor SOX2 is involved in the regulation of embryonic development, in the determination of cell fate and pluripotency maintenance activity; while the genes FGF4, LIN28, and SFRP2 have been characterized as stemness markers $[41,42]$.

Figure 4. Comparison of miRNA expression level between H9 and NT-2 cell lines upon neuronal differentiation. miRNA expression overlapping between these two cell lines is shown for up-regulated (A) and down-regulated (B) miRNAs by using of Venn diagram; (C) The quantification of the expression levels of the most significantly deregulated and overlapping miRNAs during the process of neural differentiation in H9 and NT-2 cells. Data are expressed in $\log _{2} \mathrm{FC}$ (fold change), comparing differentiated to undifferentiated cell samples. miRNAs specific for stemness were down-regulated during the differentiation process (negative fold change), whereas miRNAs specific for neuronal differentiation showed an increase of their expression in the differentiated cells (positive fold change) compared to undifferentiated cells.

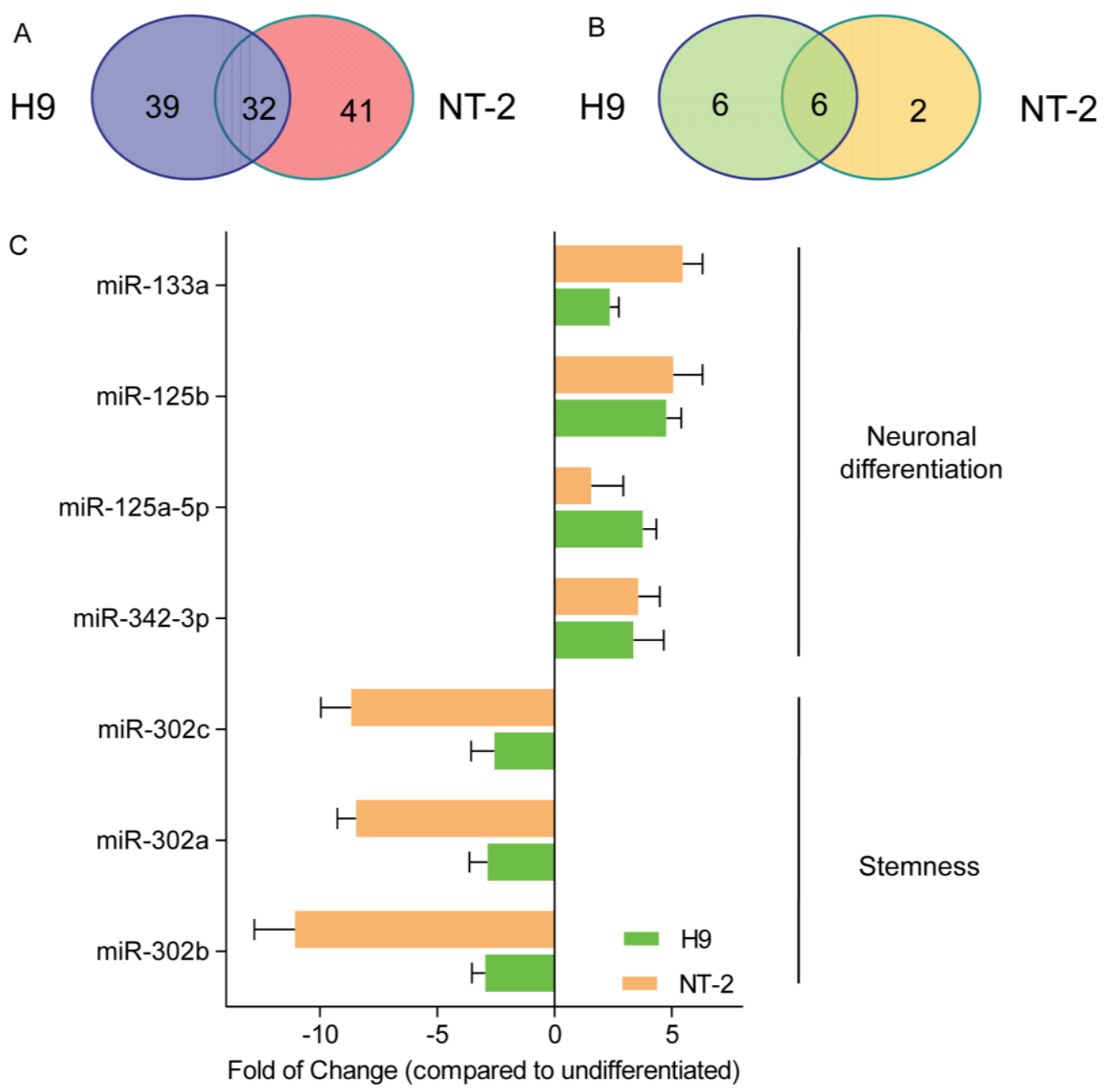




\subsection{Comparison of miRNA Expression Profiles between Neuronal Committed H9 and} Neuronal Committed NT-2 Cells

The variation in the expression of 381 miRNAs was measured by using microfluidic cards "Taqman Array MicroRNA Cards" (Applied Biosystems, Carlsbad, CA, USA) and comparing undifferentiated NT-2 and undifferentiated H9 cells (both at 1 DIV) with their respective neuronal committed counterparts, respectively at 36 DIV for NT-2 and at 10 DIV for H9.

Thirty-two miRNAs were significantly upregulated in both differentiated H9 and NT-2 cell lines (about $40 \%$ of the total up-regulated miRNAs) (Figure 4A). Six miRNAs (i.e., miR-520g, miR-518b, miR-517c, miR-302a, miR-302b, and miR-302c) were down-regulated during the differentiation process in both cell lines, representing $50 \%-75 \%$ of the total miRNAs respectively down-regulated in H9 and NT-2 cells (Figure 4B).

As expected, in both test systems we observed the down-regulation of the expression of the stemness-specific miRNA-302 family and the up-regulation of miRNAs known to be involved in the neuronal differentiation process, particularly miR-133a, miR-125 and miR-342 (Figure 4C). The obtained results indicated that these different cellular models are comparable as they are characterized by the expression of common miRNA expression profiles during the process of differentiation towards the neuronal phenotype.

\subsection{Analysis of miRNA Expression Profile Perturbations Comparing Neuronal Committed H9 and Neuronal Committed NT-2 Cells Exposed to $\mathrm{MeHgCl}$}

In order to compare the miRNA deregulation induced by $\mathrm{MeHgCl}$ exposure, which is a well-known developmental neurotoxicant, cells were treated during the initial differentiation process (see Materials and Methods, section 2.4) and the miRNA expression was analyzed by using microfluidic cards "Taqman Array MicroRNA Cards" (Applied Biosystems, Carlsbad, CA, USA). The non-cytotoxic concentrations of $\mathrm{MeHgCl}$ were chosen based on the cytotoxicity studies (Alamar Blue assay) performed on $\mathrm{H} 9$ and NT-2 cell lines. Accordingly, H9 cells were treated with $25 \mathrm{nM} \mathrm{MeHgCl}$ (between 1-10 DIV) while NT-2 were exposed to a concentration of $400 \mathrm{nM}$ (2-36 DIV). The time of exposure to $\mathrm{MeHgCl}$ differs as it was adjusted to the neuronal differentiation protocol established for these two cell lines (Figure 1). Interestingly, in $\mathrm{H} 9$ cells undergoing neuronal commitment, $\mathrm{MeHgCl}$ repeated dose exposure induced a significant up-regulation of three miRNAs, miR-183, miR-15a and miR-526b*. The miR-183 was previously found to be up-regulated in undifferentiated hESC lines [43], while miR-15a seemed to be relevant in hearth development [44]. In the case of NT-2 cell line undergoing differentiation in presence of $\mathrm{RA}, \mathrm{MeHgCl}$ treatment induced the deregulation of several miRNAs; nine of them were up-regulated (miR-141, miR-196b, miR-367, miR-302b, miR-372, miR-302a, miR-302c) and five down-regulated (miR-296, miR-217*, miR-655, miR-365, miR-181a) (Table 1). Indeed, the impairment of the differentiation process was reflected by the increased expression of stemness-related miRNAs, such as miR-302/367 cluster, miR-372 and miR-141. On the other hand, we observed the down-regulation of miRNAs, such as miR-296, miR-217, miR-655, miR-365, and miR-181a. Interestingly, amongst them, miR-296 is known to target the stemness-specific transcription factor $N A N O G$ [45] as described before [46]. Increased expression of NANOG indicates 
again the disturbance of differentiation process towards neuronal phenotype induced by $\mathrm{MeHgCl}$. Indeed, the miRNA-296 was shown to be up-regulated in mouse ESC upon RA-induced differentiation, confirming that decreased expression of $N A N O G$ is taking place during the process of neuronal differentiation [45].

Table 1. List of miRNAs up- or down-regulated upon toxic treatment with methylmercury chloride $(\mathrm{MeHgCl})$ in $\mathrm{H} 9$ and $\mathrm{NT}-2$ cell lines. $\mathrm{H} 9$ cells were exposed to $25 \mathrm{nM} \mathrm{MeHgCl}$ during the first 10 days of differentiation. NT-2 cells were exposed to $400 \mathrm{nM} \mathrm{MeHgCl}$ from 2 to $36 \mathrm{DIV}$. The fold change (FC) of miRNA expression compared to respective untreated control is indicated for each miRNA (down-regulated miRNAs are reported in italic letters).

\begin{tabular}{ccccc}
\hline Cell Line & MeHgCl Treatment & miRNA & FC & p-value \\
\hline \multirow{3}{*}{ H9 } & Time: DIV 1-10; & miR-183 & 2.82 & $<0.05$ \\
& Concentration: 25 nM & miR-15a & 19 & $<0.05$ \\
& & miR-526b* & 6.5 & $<0.05$ \\
\hline & miRNA-141 & 2.5 & $<0.05$ \\
& miRNA-196b & 3.2 & $<0.05$ \\
& miRNA-367 & 6.1 & $<0.05$ \\
& & miRNA-302b & 5.3 & $<0.05$ \\
& & miRNA-372 & 9.5 & $<0.05$ \\
& & miR-302a & 3.7 & $<0.05$ \\
& Time: DIV 2-36 & miR-302c & 3.1 & $<0.05$ \\
& Concentration: 400 nM & miR-142 & 2.4 & $<0.05$ \\
& & miR-200c & 2.3 & $<0.05$ \\
& & $m i R-296$ & -1.5 & $<0.05$ \\
& & $m i R-217 *$ & -4.4 & $<0.05$ \\
& & $m i R-655$ & -2.5 & $<0.05$ \\
& & $m i R-365$ & -1.3 & $<0.05$ \\
& & $m i R-181 a$ & -1.5 & $<0.05$ \\
\hline
\end{tabular}

3.5. Gene ontology (GO) Term Enrichment Analysis of Deregulated miRNAs Induced by the Exposure to $\mathrm{MeHgCl}$

The targets of the deregulated miRNAs in both cell lines (H9 and NT-2) have been identified by the use of miRecords database and only the ones predicted by at least five different algorithms have been used for performing GO term enrichment analysis. For these miRNAs the WEB-Gestalt toolkit (WEB-based GEne SeT AnaLysis Toolkit, Vanderbilt University, Nashville, TN, USA) [14] has been then used in order to obtain the list of the most represented GO terms and pathways, which are the potential targets of deregulated miRNAs. The most significant GO terms were defined for the up-regulated miRNAs in the NT-2 and H9 cell systems, respectively (Table 2). No overlap has been observed between the general biological processes identified in these two cell lines. 
Table 2. Enriched miRNA-biological process gene ontology (GO)-terms in NT-2 and H9 cell lines. Analysis of Gene Ontology (GO) term enrichment for gene targets of deregulated miRNAs identified in the two cell cultures (H9 and NT-2) exposed to $\mathrm{MeHgCl}$ (compared to untreated cells).

\begin{tabular}{cc}
\hline Upregulated miRNA-Biological Process GO-terms in NT-2 Cell Line & $\boldsymbol{p}$-value \\
\hline regulation of cellular macromolecule biosynthetic process (GO:2000112) & $7.70 \times 10^{-30}$ \\
regulation of cellular metabolic process (GO:0031323) & $1.02 \times 10^{-29}$ \\
regulation of cellular process (GO:0050794) & $1.27 \times 10^{-29}$ \\
regulation of macromolecule biosynthetic process (GO:0010556) & $3.83 \times 10^{-30}$ \\
regulation of macromolecule metabolic process (GO:0060255) & $3.78 \times 10^{-29}$ \\
regulation of nucleobase-containing compound metabolic process (GO:0019219) & $7.70 \times 10^{-30}$ \\
regulation of RNA metabolic process (GO:0051252) & $5.38 \times 10^{-29}$ \\
regulation of transcription from RNA polymerase II promoter (GO:0006357) & $4.63 \times 10^{-30}$ \\
regulation of transcription, DNA-dependent (GO:0006355) & $4.36 \times 10^{-29}$ \\
transcription from RNA polymerase II promoter (GO:0006366) & $3.83 \times 10^{-30}$ \\
\hline Upregulated miRNA-Biological Process GO-terms in H9 Cell Line & $p$-value \\
\hline anatomical structure morphogenesis (GO:0009653) & $2.45 \times 10^{-7}$ \\
cellular protein modification process (GO:0006464) & $2.92 \times 10^{-7}$ \\
generation of neurons (GO:0048699) & $1.58 \times 10^{-8}$ \\
nervous system development (GO:0007399) & $1.58 \times 10^{-8}$ \\
neurogenesis (GO:0022008) & $1.58 \times 10^{-8}$ \\
neuron differentiation (GO:0030182) & $2.53 \times 10^{-7}$ \\
protein modification process (GO:0036211) & $2.92 \times 10^{-7}$ \\
regulation of cell development (GO:0060284) & $1.07 \times 10^{-7}$ \\
regulation of cell differentiation (GO:0045595) & $2.69 \times 10^{-7}$ \\
regulation of developmental process (GO:0050793) & $2.69 \times 10^{-7}$ \\
\hline
\end{tabular}

Table 3. Enriched miRNA-pathways in NT-2 and H9 cell lines. Analysis of KEGG pathway enrichment for gene targets of regulated miRNAs identified in the cultures of the two cell types (H9 and NT-2) exposed to $\mathrm{MeHgCl}$ (compared to untreated cells). The overlapping pathways between H9 and NT-2 cell lines are marked in bold.

\begin{tabular}{cccc}
\hline $\begin{array}{c}\text { Upregulated miRNA-Pathways } \\
\text { in NT-2 Cell Line }\end{array}$ & $\boldsymbol{p}$-value & $\begin{array}{c}\text { Upregulated miRNA-Pathways } \\
\text { in H9 Cell Line }\end{array}$ & $\boldsymbol{p}$-value \\
\hline Axon guidance & $7.13 \times 10^{-10}$ & Cell cycle & $4.36 \times 10^{-6}$ \\
Pathways in cancer & $4.86 \times 10^{-15}$ & Axon guidance & $2.71 \times 10^{-5}$ \\
Oocyte meiosis & $3.85 \times 10^{-10}$ & TGF-beta signaling pathway & $2.46 \times 10^{-5}$ \\
MAPK signaling pathway & $3.73 \times 10^{-11}$ & p53 signaling pathway & $2.00 \times 10^{-4}$ \\
Neurotrophin signaling pathway & $3.38 \times 10^{-14}$ & Glycerophospholipid metabolism & $1.00 \times 10^{-4}$ \\
Renal cell carcinoma & $1.89 \times 10^{-8}$ & Oocyte meiosis & $8.25 \times 10^{-6}$ \\
Wnt signaling pathway & $1.70 \times 10^{-9}$ & Pathways in cancer & $1.75 \times 10^{-10}$ \\
Focal adhesion & $9.74 \times 10^{-10}$ & Neurotrophin signaling pathway & $1.22 \times 10^{-6}$ \\
Ubiquitin mediated proteolysis & $6.55 \times 10^{-9}$ & Wnt signaling pathway & $1.22 \times 10^{-6}$ \\
TGF-beta signaling pathway & $4.94 \times 10^{-9}$ & Small cell lung cancer & $1.00 \times 10^{-4}$ \\
\hline
\end{tabular}


However, interestingly, there is a strong overlap between the most enriched pathways (Table 3 in bold) identified as being under the control of the analyzed up-regulated miRNAs in both NT-2 and H9 cell lines.

Indeed six out of the ten most significantly up-regulated pathways are shared between the two tests systems. Some of these miRNAs are involved in regulation of pathways that are critical for cell-to-cell signaling during embryogenesis, such as Wnt signaling pathway, or involved in neuronal specific developmental processes, such as cell migration and neuronal differentiation, which are under the control of neurotrophin or more advanced neuronal specific maturation processes, such as the pathway involved in axonal guidance (Table 3).

\section{Discussion}

Recently in the field of regulatory toxicology, efforts have been undertaken towards more mechanistically driven approaches, aiming to improve current safety assessments of environmental chemicals, including those with neurodevelopmental potential [47].

The US National Research Council proposed a shift from the currently performed apical animal testing towards mechanistically-based toxicity pathways evaluation. It was advocated that such approaches should be based preferably on human cellular models, allowing the overcoming current shortcomings in the extrapolation between species [48,49]. In the field of adult and developmental neurotoxicity evaluation an attempt to apply the adverse outcome pathway (AOP) concept has been undertaken. The AOP framework is entirely based on mechanistic information capturing the key events from the initial molecular interaction of the toxicant with the biological target along the subsequent chain of events leading to an adverse outcome. Hopefully in the near future the AOP concept will serve as a basis for the development of testing strategies for human risk assessment [50].

In this study we have evaluated two human neuronal models derived from two different pluripotent stem cell sources, embryonic (H9 cell line) and carcinoma cells (NT-2 cell line) by comparing the expression of overlapping genes and miRNAs of undifferentiated cells versus those in the initial stage of differentiation towards neuronal phenotype. The detailed descriptions of the neuronal and glial markers characteristic of various differentiation stages of $\mathrm{H} 9$ and NT-2 cell line have been already published [26,32].

Interestingly, in this study upon neuronal differentiation an overlap between the up- (Figure 2) and down- (Figure 3) regulated genes in the human embryonic (H9) and carcinoma NT-2 cells was observed. As expected, the genes that were commonly up regulated in both models, such as COL2A1, FN1, GATA6, IAPP, and SYP were involved in the process of early embryogenesis and included $S Y P$ that is linked to neuronal differentiation. Moreover, DNMT3B, EOMES, FGF4, LIN28, SFRP2, and $S O X 2$ genes, all linked to maintenance of stemness, were commonly down-regulated in both cell models.

The presence of other genes differentially expressed in the two derived neuronal cell models, could be explained by the fact that these cell lines originate from very different types of stem cells, embryonic (H9) and carcinoma (NT-2) cells, and the neuronal differentiation protocols designed to promote their neuronal commitment are necessary different.

As miRNAs have been demonstrated to play crucial roles in nervous system development in vivo, we sought to evaluate miRNA expression profiles comparing the two neuronally committed cell 
models (NT-2 and H9). Both models underwent a significant miRNA expression regulation upon neuronal commitment. Indeed, 32 miRNAs were commonly up regulated in both models and the most significantly overexpressed were miR-133a, miR125b, miR125a-5p and miR342-3p (Figure 4), which are known to be involved in neuronal differentiation. In contrast, in both cell lines, only six miRNAs were commonly down-regulated, and all these miRNAs are known to be related to stemness maintenance. In particular, the miRNA-302 family (302c, 302a, 302b) that is known to be involved in the regulation of pluripotency maintenance, was found significantly down-regulated during the neuronal differentiation process.

Furthermore, we evaluated whether the expression of these miRNAs was deregulated after the exposure to $\mathrm{MeHgCl}$, aiming to identify possible biomarkers of in vitro induced developmental neurotoxicity. Indeed, miRNAs are supposed to regulate numerous target genes involved in the control of stress response pathways, and it could be useful to identify chemically perturbed pathways specific for neurodevelopmental toxicity.

Therefore, after repeated exposure to non-cytotoxic concentration of $\mathrm{MeHgCl}(25 \mathrm{nM})$ during the initial stage (1-10 DIV) of neural differentiation of $\mathrm{H} 9$ cell line, we identified three miRNAs that were significantly up-regulated, such as miR-183 (fold change, FC 2.8), miR-15a (FC 19) and miR-526b (FC 6.5). Interestingly, expression of the miRNA-183 and $-15 \mathrm{a}$ is linked to the non-neural differentiation processes, as miRNA-183 is highly expressed in embryonic stem cells [32] and miRNA-15a is involved in cardiac developmental differentiation. In the case of NT-2 cell line exposed to $\mathrm{MeHgCl} 400 \mathrm{nM}$, a significant up-regulation of the stemness related miRNAs was found (miR-302b, miR-367 and miR-372) suggesting that the process of neuronal differentiation could be affected. In agreement with this observation, we detected the down-regulation of the miRNA-296 (FC 1.5), which is known to inhibit the expression of the stemness-related transcription factor NANOG [45]. We also observed down-regulation of miRNA-217 (FC 4.4), miRNA-655 (FC 2.5), miRNA -365 (FC 1.3) and miR-181a (FC 1.5), the involvement of which in cell differentiation or stemness phenotype maintenance was no previously observed.

Interestingly, we did not observe any overlap between the deregulated miRNAs (up or down) determined in the $\mathrm{H} 9$ compared with $\mathrm{NT}-2$ cell line exposed to $\mathrm{MeHgCl}$. This may be explained by the fact that different protocols of cell differentiation had been applied and/or that these cellular models are derived from very diverse cellular sources (embryonic vs. carcinogenic). However, the different concentrations of toxicant were deliberately selected based on cytotoxicity assays (first non-cytotoxic concentration) and different time of exposure was selected based on the protocols of neuronal differentiation (see Materials and Methods).

Furthermore, we performed GO terms enrichment analysis to identify the most represented biological processes and cellular pathways controlled by the miRNAs that were perturbed upon the exposure to $\mathrm{MeHgCl}$. The biological processes that were under the control of the up-regulated miRNAs in NT-2 cell line were mainly correlated with the regulation of metabolic and macromolecule biosynthetic cellular processes, as well regulation of DNA-dependent transcription from RNA polymerase II promoter. Interestingly, the biological processes identified in the H9 cell line that were under control of the deregulated miRNAs were linked to neuronal specific developmental processes such as generation of neurons, nervous system development, neurogenesis, or regulation of developmental processes. Moreover, GO terms analysis for the most significantly up-regulated miRNAs in H9 and 
NT-2 cells did not determine any overlap between the biological processes identified in these two cell lines. These results would suggest that at an early stage of differentiation, exposure to $\mathrm{MeHgCl}$ affected mainly the specific neurodevelopmental process of neural precursor cells derived from the $\mathrm{H} 9$ cell line. In the case of the NT-2 cell line exposed to the same toxicant more general biological processes were affected, possibly due to a delay in neural differentiation when compared to H9 cell line. However, interestingly, NT-2 and H9 cell lines exposed to $\mathrm{MeHgCl}$ displayed a strong overlap of the most enriched pathways (Table 3), identified as being under the control of up-regulated miRNAs. Indeed, six out of the ten most significant pathways involved in the response to $\mathrm{MeHgCl}$ treatment are shared between the two test systems. Some of these common miRNA-targeted pathways could play a role in critical developmental processes, specific for neuronal differentiation, such as axonal guidance or neurotrophin-related signaling pathways. These results suggest that similar pathways are affected by $\mathrm{MeHgCl}$ exposure in both neural cultures derived from NT-2 or H9 (Table 3) and, most importantly, these pathways are specifically linked to neuronal cell function regulations. These comparative studies allowed us to conclude that miRNA deregulation induced by the exposure to $\mathrm{MeHgCl}$ perturbed similar neuronally related pathways in both $\mathrm{H} 9$ and NT-2 derived neuronal cultures, which might be consequentially identified as toxicity pathways.

Although further investigation is necessary, the obtained results suggest that miRNA expression profiling could be used as a promising tool for simplified functional evaluation of the toxicity pathways involved in developmental neurotoxicity mechanisms and could improve predictive human toxicity testing, especially when human in vitro models are applied. However, taking into consideration the peculiar nature of pluripotent stem cells, a high level of standardization of undifferentiated and differentiated cell cultures is required [29] in order to ensure the robustness and reproducibility of results that in the future might serve for regulatory toxicology purposes.

\section{Conclusions}

The miRNA expression profiles have been compared during the early phase of neural commitment of the human pluripotent carcinoma (NT-2 cell line) and embryonic stem cells (H9 cell line). miRNas signatures were analyzed upon early stage of neural cell differentiation in the control cultures and after exposure to a repeated administration of $\mathrm{MeHgCl}$ at non-cytotoxic concentrations. The results obtained from this comparative study showed that miRNA deregulation induced by the exposure to $\mathrm{MeHgCl}$ perturbed similar pathways that were linked to specific neuronal differentiation processes in both cultures (derived from H9 or NT-2 cell lines). Comparison of miRNA profiles in these two in vitro DNT models strengthens the consideration to use this approach for identification and functional evaluation of toxicity pathways involved in developmental neurotoxicity mechanisms. Therefore miRNA profiling could represent a novel toxicological tool for detecting and characterizing human DNT hazards.

\section{Acknowledgments}

This work was financially supported by the Institute for Health and Consumer Protection of the European Commission Joint Research Centre (Ispra, Italy). 
Marco Fabbri is a student of the Ph.D. program in Biotechnology, School of Biological and Medical Sciences, University of Insubria, Varese, Italy.

\section{Author Contributions}

Giorgia Pallocca and Anna Bal-Price conceived, designed the experiments and wrote the paper; Giorgia Pallocca, Silvia Nerini-Molteni, Francesca Pistollato, Dimitra, Zagura, Maria Grazia Sacco performed the experiments; Laura Gribaldo, Susanne Bremer-Hoffmann and Marco Fabbri analyzed the data and contributed to the data interpretation.

\section{Conflicts of Interest}

The authors declare no conflict of interest.

\section{References}

1. Christensen, M.; Schratt, G.M. microRNA involvement in developmental and functional aspects of the nervous system and in neurological diseases. Neurosci. Lett. 2009, 466, 55-62.

2. Krichevsky, A.M.; King, K.S.; Donahue, C.P.; Khrapko, K.; Kosik, K.S. A microRNA array reveals extensive regulation of microRNAs during brain development. RNA 2003, 9, 1274-1281.

3. Miska, E.A.; Alvarez-Saavedra, E.; Townsend, M.; Yoshii, A.; Sestan, N.; Rakic, P.; Constantine-Paton, M.; Horvitz, H.R. Microarray analysis of microRNA expression in the developing mammalian brain. Genome Biol. 2004, 5, R68.

4. Krek, A.; Grun, D.; Poy, M.N.; Wolf, R.; Rosenberg, L.; Epstein, E.J.; MacMenamin, P.; da Piedade, I.; Gunsalus, K.C.; Stoffel, M.; et al. Combinatorial microRNA target predictions. Nat. Genet. 2005, 37, 495-500.

5. Kim, J.; Inoue, K.; Ishii, J.; Vanti, W.B.; Voronov, S.V.; Murchison, E.; Hannon, G.; Abeliovich, A. A MicroRNA feedback circuit in midbrain dopamine neurons. Science 2007, 317, 1220-1224.

6. De Pietri Tonelli, D.; Pulvers, J.N.; Haffner, C.; Murchison, E.P.; Hannon, G.J.; Huttner, W.B. miRNAs are essential for survival and differentiation of newborn neurons but not for expansion of neural progenitors during early neurogenesis in the mouse embryonic neocortex. Development 2008, 135, 3911-3921.

7. Giraldez, A.J.; Cinalli, R.M.; Glasner, M.E.; Enright, A.J.; Thomson, J.M.; Baskerville, S.; Hammond, S.M.; Bartel, D.P.; Schier, A.F. MicroRNAs regulate brain morphogenesis in zebrafish. Science 2005, 308, 833-838.

8. Delaloy, C.; Liu, L.; Lee, J.A.; Su, H.; Shen, F.; Yang, G.Y.; Young, W.L.; Ivey, K.N.; Gao, F.B. MicroRNA-9 coordinates proliferation and migration of human embryonic stem cell-derived neural progenitors. Stem Cell 2010, 6, 323-335.

9. Maller Schulman, B.R.; Liang, X.; Stahlhut, C.; DelConte, C.; Stefani, G.; Slack, F.J. The let-7 microRNA target gene, Mlin41/Trim71 is required for mouse embryonic survival and neural tube closure. Cell Cycle 2008, 7, 3935-3942. 
10. Makeyev, E.V.; Zhang, J.; Carrasco, M.A.; Maniatis, T. The MicroRNA miR-124 promotes neuronal differentiation by triggering brain-specific alternative pre-mRNA splicing. Mol. Cell 2007, 27, 435-448.

11. Schratt, G. Fine-tuning neural gene expression with microRNAs. Curr. Opin. Neurobiol. 2009, 19, 213-219.

12. Vo, N.; Klein, M.E.; Varlamova, O.; Keller, D.M.; Yamamoto, T.; Goodman, R.H.; Impey, S. A cAMP-response element binding protein-induced microRNA regulates neuronal morphogenesis. Proc. Natl. Acad. Sci. USA 2005, 102, 16426-16431.

13. Konopka, W.; Kiryk, A.; Novak, M.; Herwerth, M.; Parkitna, J.R.; Wawrzyniak, M.; Kowarsch, A.; Michaluk, P.; Dzwonek, J.; Arnsperger, T.; et al. MicroRNA loss enhances learning and memory in mice. J. Neurosci. 2010, 30, 14835-14842.

14. Wang, J.; Duncan, D.; Shi, Z.; Zhang, B. WEB-based GEne SeT AnaLysis Toolkit (WebGestalt): Update 2013. Nucleic Acids Res. 2013, 41, W77-W83.

15. Kaur, P.; Armugam, A.; Jeyaseelan, K. MicroRNAs in neurotoxicity. J. Toxicol. 2012, 2012, 870150, doi:10.1155/2012/870150.

16. Lee, Y.; Samaco, R.C.; Gatchel, J.R.; Thaller, C.; Orr, H.T.; Zoghbi, H.Y. miR-19, miR-101 and miR-130 co-regulate ATXN1 levels to potentially modulate SCA1 pathogenesis. Nat. Neurosci. 2008, 11, 1137-1139.

17. Nelson, P.T.; Wang, W.X. MiR-107 is reduced in Alzheimer's disease brain neocortex: Validation study. J. Alzheimer's Dis. 2010, 21, 75-79.

18. Grandjean, P.; Landrigan, P.J. Developmental neurotoxicity of industrial chemicals. Lancet 2006, 368, 2167-2178.

19. Hogberg, H.T.; Kinsner-Ovaskainen, A.; Coecke, S.; Hartung, T.; Bal-Price, A.K. mRNA expression is a relevant tool to identify developmental neurotoxicants using an in vitro approach. Toxicol. Sci. 2010, 113, 95-115.

20. Hogberg, H.T.; Kinsner-Ovaskainen, A.; Hartung, T.; Coecke, S.; Bal-Price, A.K. Gene expression as a sensitive endpoint to evaluate cell differentiation and maturation of the developing central nervous system in primary cultures of rat cerebellar granule cells (CGCs) exposed to pesticides. Toxicol. Appl. Pharmacol. 2009, 235, 268-286.

21. Claudio, L.; Kwa, W.C.; Russell, A.L.; Wallinga, D. Testing methods for developmental neurotoxicity of environmental chemicals. Toxicol. Appl. Pharmacol. 2000, 164, 1-14.

22. Eriksson, P. Developmental neurotoxicity of environmental agents in the neonate. Neurotoxicology 1997, 18, 719-726.

23. Rodier, P.M. Developing brain as a target of toxicity. Environ. Health Perspect. 1995, 103, 73-76.

24. Tilson, H.A. Neurotoxicology risk assessment guidelines: Developmental neurotoxicology. Neurotoxicology 2000, 21, 189-194.

25. Buzanska, L.; Sypecka, J.; Nerini-Molteni, S.; Compagnoni, A.; Hogberg, H.T.; del Torchio, R.; Domanska-Janik, K.; Zimmer, J.; Coecke, S. A human stem cell-based model for identifying adverse effects of organic and inorganic chemicals on the developing nervous system. Stem Cells 2009, 27, 2591-2601. 
26. Stummann, T.C.; Hareng, L.; Bremer, S. Hazard assessment of methylmercury toxicity to neuronal induction in embryogenesis using human embryonic stem cells. Toxicology 2009, 257, 117-126.

27. Balmer, N.V.; Weng, M.K.; Zimmer, B.; Ivanova, V.N.; Chambers, S.M.; Nikolaeva, E.; Jagtap, S.; Sachinidis, A.; Hescheler, J.; Waldmann, T.; et al. Epigenetic changes and disturbed neural development in a human embryonic stem cell-based model relating to the fetal valproate syndrome. Hum. Mol. Genet. 2012, 21, 4104-4114.

28. Klaric, M.; Winkler, J.; Vojnits, K.; Meganathan, K.; Jagtap, S.; Ensenat-Waser, R.; Hescheler, J.; Sachinidis, A.; Bremer-Hoffmann, S. Current status of human pluripotent stem cell based in vitro toxicity tests. Front. Biosci. Schol. Ed. 2013, 5, 118-133.

29. Pistollato, F.; Bremer-Hoffmann, S.; Healy, L.; Young, L.; Stacey, G. Standardization of pluripotent stem cell cultures for toxicity testing. Expert Opin. Drug Metab. Toxicol. 2012, 8, 239-257.

30. Scott, C.W.; Peters, M.F.; Dragan, Y.P. Human induced pluripotent stem cells and their use in drug discovery for toxicity testing. Toxicol. Lett. 2013, 219, 49-58.

31. Bosnjak, Z.J. Developmental neurotoxicity screening using human embryonic stem cells. Exp. Neurol. 2012, 237, 207-210.

32. Laurenza, I.; Pallocca, G.; Mennecozzi, M.; Scelfo, B.; Pamies, D.; Bal-Price, A. A human pluripotent carcinoma stem cell-based model for in vitro developmental neurotoxicity testing: Effects of methylmercury, lead and aluminum evaluated by gene expression studies. Int. J. Dev. Neurosci. 2013, 31, 679-691.

33. Hartley, R.S.; Margulis, M.; Fishman, P.S.; Lee, V.M.; Tang, C.M. Functional synapses are formed between human NTera2 (NT-2N, hNT) neurons grown on astrocytes. J. Comp. Neurol. 1999, 407, 1-10.

34. Serra, M.; Leite, S.B.; Brito, C.; Costa, J.; Carrondo, M.J.; Alves, P.M. Novel culture strategy for human stem cell proliferation and neuronal differentiation. J. Neurosci. Res. 2007, 85, 3557-3566.

35. Pistollato, F.; Louisse, J.; Scelfo, B.; Mennecozzi, M.; Accordi, B.; Basso, G.; Gaspar, A.J.; Zagoura, D.; Barilari, M.; Palosaari, T.; et al. Development of a pluripotent stem cell derived neuronal model to identify chemically induced pathway perturbations in relation to neurotoxicity: Effects of CREB pathway inhibition. Toxicol. Appl. Pharmacol. 2014, doi:10.1016/j.taap.2014.08.007, in press.

36. O'Brien, J.; Wilson, I.; Orton, T.; Pognan, F. Investigation of the Alamar Blue (resazurin) fluorescent dye for the assessment of mammalian cell cytotoxicity. Eur. J. Biochem. 2000, 267, 5421-5426.

37. Gentleman, R.C.; Carey, V.J.; Bates, D.M.; Bolstad, B.; Dettling, M.; Dudoit, S.; Ellis, B.; Gautier, L.; Ge, Y.; Gentry, J.; et al. Bioconductor: Open software development for computational biology and bioinformatics. Genome Biol. 2004, 5, R80.

38. Dvinge, H.; Bertone, P. HTqPCR: High-throughput analysis and visualization of quantitative real-time PCR data in R. Bioinformatics 2009, 25, 3325-3326.

39. Smyth, G.K. Linear models and empirical bayes methods for assessing differential expression in microarray experiments. Stat. Appl. Genet. Mol. Biol. 2004, 3, doi:10.2202/1544-6115.1027. 
40. Xiao, F.; Zuo, Z.; Cai, G.; Kang, S.; Gao, X.; Li, T. miRecords: An integrated resource or microRNA-target interactions. Nucleic Acids Res. 2009, 37, D105-D110.

41. Nichols, J.; Zevnik, B.; Anastassiadis, K.; Niwa, H.; Klewe-Nebenius, D.; Chambers, I.; Schöler, H.; Smith, A. Formation of pluripotent stem cells in the mammalian embryo depends on the POU transcription factor Oct4. Cell 1998, 95, 379-391.

42. Richards, M.; Tan, S.P.; Tan, J.H.; Chan, W.K.; Bongso, A. The transcriptome profile of human embryonic stem cells as defined by SAGE. Stem Cells 2004, 22, 51-64.

43. Lavon, I.; Zrihan, D.; Granit, A.; Einstein, O.; Fainstein, N.; Cohen, M.A.; Zelikovitch, B.; Shoshan, Y.; Spektor, S.; Reubinoff, B.E.; et al. Gliomas display a microRNA expression profile reminiscent of neural precursor cells. Neuro-oncology 2010, 12, 422-433.

44. Porrello, E.R.; Johnson, B.A.; Aurora, A.B.; Simpson, E.; Nam, Y.J.; Matkovich, S.J.; Dorn, G.W., 2nd; van Rooij, E.; Olson, E.N. MiR-15 family regulates postnatal mitotic arrest of cardiomyocytes. Circ. Res. 2011, 109, 670-679.

45. Tay, Y.; Zhang, J.; Thomson, A.M.; Lim, B.; Rigoutsos, I. MicroRNAs to Nanog, Oct4 and SOX2 coding regions modulate embryonic stem cell differentiation. Nature 2008, 455, 1124-1128.

46. Pallocca, G.; Fabbri, M.; Sacco, M.G.; Gribaldo, L.; Pamies, D.; Laurenza, I.; Bal-Price, A. miRNA expression profiling in a human stem cell-based model as a tool for developmental neurotoxicity testing. Cell Biol. Toxicol. 2013, 29, 239-257.

47. Bal-Price, A.; Coecke, S.; Costa, L.; Crofton, K.M.; Fritsche, E.; Goldberg, A.; Grandjean, P.; Lein, P.J.; Li, A.; Lucchini, R.; et al. Advancing the science of developmental neurotoxicity (DNT): Testing for better safety evaluation. Altex 2012, 29, 202-215.

48. Leist, M.; Hartung, T. Inflammatory findings on species extrapolations: Humans are definitely no 70-kg mice. Altex 2013, 30, 227-230.

49. Paparella, M.; Daneshian, M.; Hornek-Gausterer, R.; Kinzl, M.; Mauritz, I.; Muhlegger, S. Uncertainty of testing methods - What do we (want to) know? Altex 2013, 30, 131-144.

50. Bal-Price, A.; Crofton, K.M.; Shafer, T.; Sachana, M.; Behl, M.; Forsby, A.; Hargreaves, A.; Landesmann, B.; Lein, P.J.; Louisse, J.; et al. Workshop report: Adverse outcome pathways (AOP) relevant to neurotoxicity critical rev in toxicology. Crit. Rev. Toxicol. 2014, submitted for publication.

(C) 2014 by the authors; licensee MDPI, Basel, Switzerland. This article is an open access article distributed under the terms and conditions of the Creative Commons Attribution license (http://creativecommons.org/licenses/by/3.0/). 